\title{
Relationship between COVID-19 infection and neurodegeneration: Computational insight into interactions between the SARS-CoV-2 spike protein and the monoamine oxidase enzymes
}

\author{
Lucija Hok, ${ }^{1}$ Hrvoje Rimac, ${ }^{2}$ Janez Mavri ${ }^{3}$ and Robert Vianello ${ }^{1, *}$
}

\author{
${ }^{1}$ Laboratory for the Computational Design and Synthesis of Functional Materials, Division of Organic \\ Chemistry and Biochemistry, Ruđer Bošković Institute, Zagreb, Croatia. \\ ${ }^{2}$ Department of Medicinal Chemistry, University of Zagreb Faculty of Pharmacy and Biochemistry, Zagreb, \\ Croatia. \\ ${ }^{3}$ Laboratory for Biocomputing and Bioinformatics, National Institute of Chemistry, Ljubljana, Slovenia. \\ * Corresponding author. Email: robert.vianello@irb.hr
}

ABSTRACT: Although COVID-19 has been primarily associated with pneumonia, recent data show that its causative agent, the SARS-CoV-2 coronavirus, can infect many vital organs beyond the lungs, including the heart, kidneys and the brain. The literature agrees that COVID-19 is likely to have long-term mental health effects on infected individuals, which signifies a need to understand the role of the virus in the pathophysiology of brain disorders that is currently unknown and widely debated. Our docking and molecular dynamic simulations show that the affinity of the spike protein from the wild type (WT) and the South African B.1.351 (SA) variant towards the MAO enzymes is comparable to that for its ACE2 receptor.

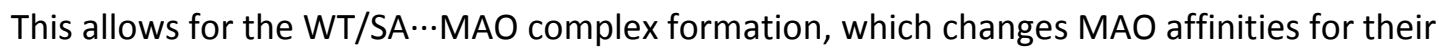
neurotransmitter substrates, thus consequently impacting the rates of their metabolic conversion and misbalancing their levels. Knowing that this fine regulation is strongly linked with the etiology of various brain pathologies, these results are the first to highlight the possibility that the interference with the brain MAO catalytic activity is responsible for the increased neurodegenerative illnesses following a COVID-19 infection, thus placing a neurobiological link between these two conditions in the spotlight. Since the obtained insight suggests that a more contagious SA variant causes even larger disturbances, and with new and more problematic strains likely emerging in the near future, we firmly advise that the presented prospect of the SARS-CoV-2 induced neurological complications should not be ignored, but rather requires further clinical investigations to achieve an early diagnosis and timely therapeutic interventions.

KEYWORDS: brain amines $\bullet$ COVID-19 • molecular dynamic simulations $\bullet$ neurodegeneration $\bullet$ neurotransmitters $\bullet$ SARS-CoV-2 • South African B.1.351 variant • spike protein 
bioRxiv preprint doi: https://doi.org/10.1101/2021.08.30.458208; this version posted August 31, 2021. The copyright holder for this preprint (which was not certified by peer review) is the author/funder, who has granted bioRxiv a license to display the preprint in perpetuity. It is made available under aCC-BY-NC-ND 4.0 International license.

\section{TABLE OF CONTENTS ENTRY}

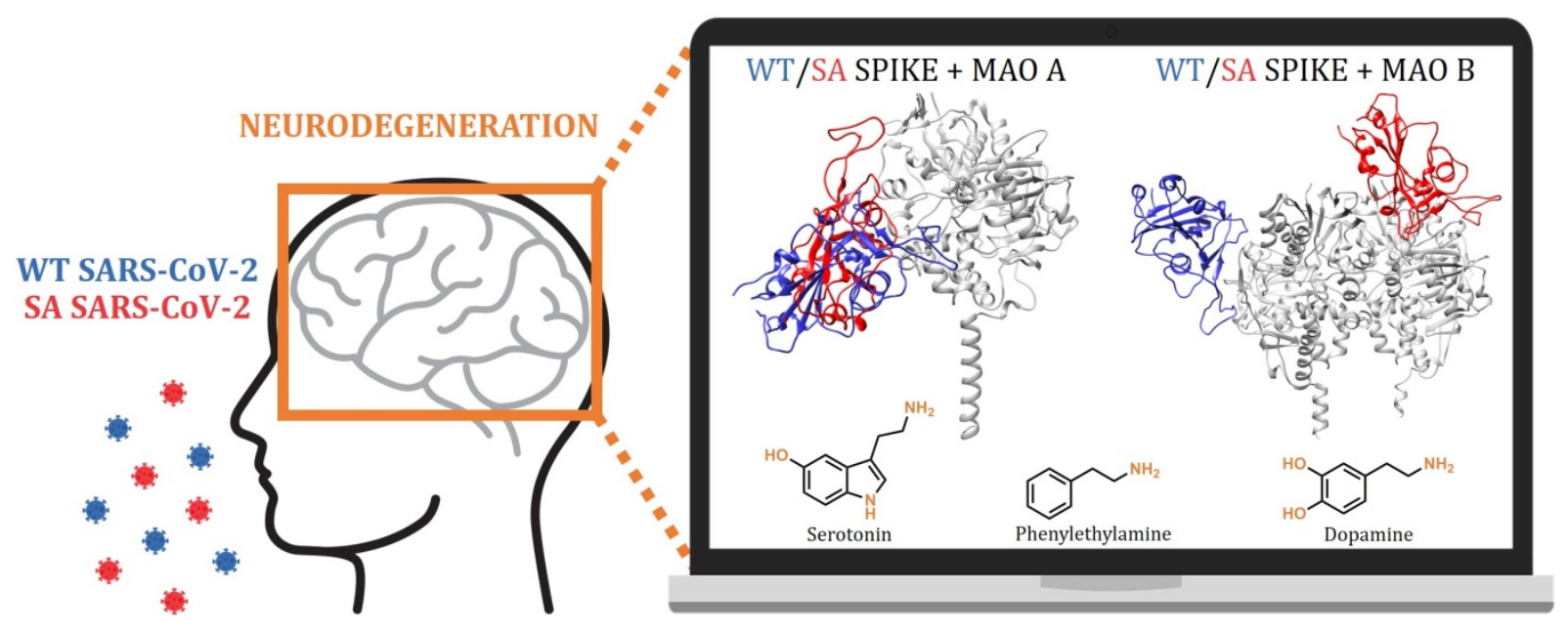

Docking and molecular dynamic simulations highlight the possibility that the interference with the brain monoamine oxidase (MAO) catalytic activity is responsible for the increased neurodegenerative illnesses following a COVID-19 infection. 


\section{INTRODUCTION}

In December 2019, a novel SARS-CoV-2 coronavirus emerged from China and spread worldwide as a pandemic, causing a public health emergency and killing over 2 million people in the first year,[1] while totalling over 4.5 million fatal outcomes by now (August 2021).[2] This infection is responsible for heterogeneous clinical disturbances, leading to severe pneumonia and the acute respiratory distress syndrome, termed COVID-19, which manifests not only as a respiratory illness but also impacts the cardiovascular, renal, and the nervous system functions.[3] Until now, this outbreak has been accompanied by a high burden on a lot of social, economic and political distress throughout the world[4] due to governmental measures of containment such as quarantine, social distancing, and lockdown. Importantly, the long-term consequences of the virus, including its effects on mental and physical health, however, might even pose a much more serious threat in the years to come.

Despite the fact that coronaviruses have not yet been linked with particular long-term neurological sequels, the occurrence of these manifestations in COVID-19 patients is becoming increasingly reported.[57] Although this suggests a possibly acute or a subacute neuropathogenicity of the virus, the risk of neurological complications in patients affected by the SARS-CoV-2 is still not entirely clarified, $[6,8,9]$ and should not be ignored.

The SARS-CoV-2 is a novel virus and its pathophysiological mechanisms in various physiological systems are yet to be fully understood. However, a lot can be learnt from the other coronavirus subtypes known to infect humans.[8] A great structural similarity between the SARS-CoV-2 and beta coronaviruses suggests a hypothesis that the SARS-CoV-2 also possesses similar neurotrophic and neuroinvasive properties. Additionally, the SARS-CoV and the SARS-CoV-2 share around 80\% genome similarity[10] and use the same ACE2 host receptor to infiltrate human cells.[11] Apart from this role of the ACE2 receptor, gene expression studies have revealed that the ACE2 gene shows the most significant co-expression and coregulation with the aromatic $L$-amino acid decarboxylase, which is responsible for the biosynthesis of biogenic amines and the conversion of L-DOPA into dopamine. This indicates that ACE2 downregulation, induced by the SARS-CoV-2 infection, might be associated with concomitant alterations in the brain amine levels, which is strongly implicated in the etiology of Alzheimer and Parkinson diseases.[12] In addition, the CT/MRI scan of COVID-19 patients showed an acute necrotizing encephalopathy, a rare encephalopathy typically associated with a viral infection of the brain tissue,[13] indicating a direct CNS infection by the SARS-CoV-2. In fact, at least four known coronaviruses (HCoV-229E, HCoV-OC43, SARS-CoV, and MERS-CoV) are able to penetrate the central nervous system,[14] and the literature agrees that the CNS infection by the SARS-CoV-2 virus may promote a development of neurodegenerative disease,[15-17] especially in people already at risk.[18] Still, a significant difference between the SARS-CoV-2 and other coronaviruses is the longer length of the spike protein sequence.[19] This disparity has been suggested to confer a higher transmissibility potential to the SARS-CoV-2, making it possible for the virus to infect humans of different races and geographical origins.[19-21] It is proposed that the virus enters the CNS through different routes, 
bioRxiv preprint doi: https://doi.org/10.1101/2021.08.30.458208; this version posted August $31,2021$. The copyright holder for this preprint (which was not certified by peer review) is the author/funder, who has granted bioRxiv a license to display the preprint in perpetuity. It is made available under aCC-BY-NC-ND 4.0 International license.

including the olfactory and trigeminal nerves, the cerebrospinal fluid, the vasculature, and the lymphatic system,[22] even without an initial lung involvement. Once the virus enters the nervous system, it can bind to the highly expressed ACE2 receptor in glial cells and neurons, and from there disseminate throughout the brain.

Clinical studies show that approximately $36 \%$ of all COVID-19 patients exhibit neurological symptoms such as stroke, headache, impaired consciousness, and paresthesia.[23] COVID-19 patients can also exhibit neurobehavioral symptoms such as euphoria, anxiety, and depression, as well as cognitive dysfunction, especially in elderly patients, which are the most susceptible to the infection.[24] Accumulated evidence confirms the SARS-CoV-2 potential to invade the CNS, however, its effects at the molecular and mechanistic levels have so far only been speculations and hypotheses. Although a COVID-19 infection certainly represents a stressful event, which, on its own, may have a role in triggering neurodegeneration,[25] in this work we used a range of computational approaches to demonstrate that the SARS-CoV-2 can trigger misbalances in the monoaminergic system by binding the monoamine oxidase enzymes, MAO A and MAO B, with affinities comparable to those for its ACE2 receptor, thus causing a significant dysregulation in the way MAOs interact with their physiological substrates. Since MAO enzymes are involved in the metabolic clearance and regulation of brain amine levels,[26] including neurotransmitters dopamine and serotonin, whose disparity is strongly linked to the etiology and course of various neurological illnesses, $[27,28]$ such a downregulation and modified MAO activity likely represent incipient stages of neurological disturbances, which are already broadly speculated in the literature.[29-32] Importantly, a potential relationship between the MAO enzymes and a SARS-CoV-2 infection has recently been proposed by Cuperlovic-Culf, Green and co-workers,[33] who used metabolomic profiling to detect a decrease in the concentration of phenylethylamine (PEA) metabolites within the cerebrospinal fluid and blood of COVID-19 related patients relative to healthy individuals, a trend similarly observed with more than 200 other metabolites, involving amino acids and their derivatives.[34] Knowing that MAO B preferentially degrades PEA in the CNS,[26] the authors ascribed this observation to a possible interference of the spike protein with the substrate entrance to the MAO B active site, thus providing a justification to our hypothesis. Additionally, it allows us to be confident that our work aids in identifying the critical role of the MAO enzymes towards an increased incidence of neurological disorders in the SARS-CoV-2 infected individuals, therefore placing a neurobiological link between these two conditions in the spotlight. 


\section{RESULTS AND DISCUSSION}

\section{Interactions between the ACE2 receptor and the spike protein from the WT SARS-CoV-2 and its South}

\section{African variant B.1.351}

The SARS-CoV-2 infiltrates human cells through an interaction between the virus S1 spike protein and the ACE2 receptor, a mechanism that has been extensively studied and characterized using various structural[35,36] and computational[37-39] techniques. Therefore, we felt it was useful to employ our computational setup to find relevant binding poses and dynamical features of the spike protein-ACE2 complexes and benchmark the obtained results with relevant literature data. By doing so, we have considered the wild-type (WT) virus and its B.1.351 South African (SA) variant, which is known to possess a higher ACE2 binding affinity,[40] an increased transmissibility and infectivity, and more severe clinical outcomes, $[40,41]$ all of which make it a good model to discuss relative differences among strains. Therefore, after a docking analysis had suggested relevant binding poses as starting points for the molecular dynamic simulations, the latter identified a representative structure of the WT $\cdots A C E 2$ complex (Figure S1) that closely matches crystal structures.[35,36] Importantly, the subsequent MM-GBSA analysis predicted a binding free energy among proteins $\Delta G_{\mathrm{BIND}}=-46.6 \mathrm{kcal} \mathrm{mol}^{-1}$, being in excellent agreement with $-46.4 \mathrm{kcal} \mathrm{mol}^{-1}$ independently reported by Yarovski,[42] Murugan[43] and their co-workers, which served as a reference. Also, a decomposition of the binding affinity on a per-residue basis underlined crucial residues in both proteins that are contributing the most to the binding (Table S1). Interestingly, the top 15 spike protein residues are responsible for around $78 \%$ of the total binding energy, and all belong to the receptor-binding motif (RBM) of the receptor-binding domain (RBD), in line with other reports, $[35,36,44]$ which confirms the validity of our calculations. The only exception is Lys417 with a notable contribution of $-0.95 \mathrm{kcal} \mathrm{mol}^{-1}$, which forms a salt bridge with Asp30 from ACE2, as demonstrated earlier.[35,36,42] Also, within residues disfavouring the binding, one notices that the first two residues, Asp405 (0.99 kcal mol$\left.{ }^{-1}\right)$ and Glu406 (0.80 $\left.\mathrm{kcal} \mathrm{mol}^{-1}\right)$, do not belong to the spike protein RBM area, while the third one, Glu484 $\left(0.66 \mathrm{kcal} \mathrm{mol}^{-1}\right)$, is one of the residues that is mutated in the SA variant to Lys484, where it exhibits a reduced unfavourable contribution by $0.13 \mathrm{kcal} \mathrm{mol}^{-1}$, from $0.66 \mathrm{kcal} \mathrm{mol}^{-1}$ in WT to $0.53 \mathrm{kcal} \mathrm{mol}^{-1}$ in SA (Table S1). Also, a highly unfavourable contribution from Ser19 in ACE2 $\left(+2.42 \mathrm{kcal} \mathrm{mol}^{-1}\right)$ agrees with the reported virus ability to improve binding upon changing the nearby environment.[45]

To put these values into a context, let us discuss data for a more contagious B.1.351 SA variant, first identified in South Africa in October 2020.[46] It carries the N501Y, E484K and K417N mutations in the RBD area[47] that confer an increased antibody resistance.[48] The overlap between the binding poses (Figure S2) does not reveal any significant difference in the way the SA variant approaches ACE2 relative to the WT, yet the identification of specific residues governing the interaction shows insightful aspects. Similarly to the WT, in the SA variant all of the top contributing residues belong, without exceptions, to the RBM area. Still, to our surprise, the most dominant residue is Tyr501, which is mutated from Asn501 in WT. Besides, its individual contribution of $-9.11 \mathrm{kcal} \mathrm{mol}^{-1}$ surpasses all WT residues and is solely responsible for around 
$17 \%$ of the total binding energy. Specifically, through the N501Y mutation, the SARS-CoV-2 increases the individual contribution of this residue by as much as $-6.7 \mathrm{kcal} \mathrm{mol}^{-1}$, which is both highly significant and highly disturbing, knowing that this mutation is well conserved and present in the UK and Brazilian strains as well,[47] although most likely independently evolved.[49] The reasons for the increased Tyr501 contribution are threefold; Tyr501 forms (i) $\mathrm{O}-\mathrm{H} \cdots \mathrm{O}$ hydrogen bonds with Asp38, (ii) cation $\cdots \pi$ interactions with Lys353, and (iii) T-shaped C-H $\cdots \pi$ contacts with Tyr41, which is, amazingly, exactly the same binding environment demonstrated in the UK variant,[50] where this is the only RBM mutation. Overall, this results in a significantly higher binding affinity of the SA strain towards ACE2, $\Delta G_{\text {BIND }}=-54.8 \mathrm{kcal} \mathrm{mol}^{-1}$, and directly translates to its higher infectiveness, strongly coupled features well-demonstrated across species.[38] At this point, it is worth to stress that $\Delta G_{\text {BIND }}$ values obtained by this approach are somewhat overestimated in absolute terms. This is a known limitation of the employed MM-GBSA approach, as extensively discussed in a recent review by Homeyer and Gohlke,[51] which also underlined its huge potential in predicting relative binding energies in biomolecular complexes $[39,42,43,51]$, precisely how this approach was used and discussed here. In this context, our analysis successfully reproduced the higher affinity of the SA strain, being in excellent agreement with experimental data,[40] thus further validating our computational setup. It is also interesting to observe that, despite the three mutations in the RBD area, the order of contributing residues is mostly unchanged among strains, which underlines the significance of single point mutations within this structural element and raises concerns that further mutations might likely offer even more problematic SARS-CoV-2 variants. Overall, we can summarize that, through the three mutations (N501Y, E484K, K417N), the SA variant increases its ACE2 affinity by $-5.8 \mathrm{kcal} \mathrm{mol}^{-1}$, being solely responsible for almost $70 \%$ of the overall affinity gain $\left(-8.2 \mathrm{kcal} \mathrm{mol}^{-1}\right)$ between the SA strain and ACE2. This strongly confirms the hypothesis that positively selected virus mutations convey benefits regarding immune evasion and viral fitness, but also for the ACE2 binding, thus contributing to the evolution rate and expectedly causing higher disturbances in the infected organisms.

\section{Interactions between the MAO enzymes (MAO A and MAO B) and the spike protein from the WT SARS-}

\section{CoV-2 virus and its South African variant B.1.351}

After establishing that the WT and SA strains recognize ACE2 in almost identical ways, mainly through their

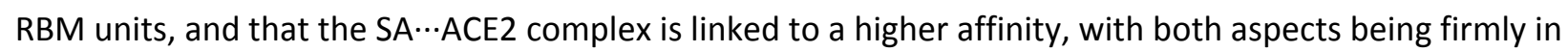
line with experimental data, we proceeded by applying the same protocol for the interaction among strains and the MAO isoforms. In each case, docking analysis elucidated ten most favourable binding poses (Figure S3), which were submitted to MD simulations (for details, see Computational Details), and trajectories with the highest protein-protein affinities were used for further analysis. Elucidated representative structures are shown in Figures S4 and S5, while the calculated affinities and their decomposition on a per-residue basis are given in Tables S2 and S3. In addition, the overlap of the resulting spike protein binding poses to each MAO isoform is depicted in Figure 1. 

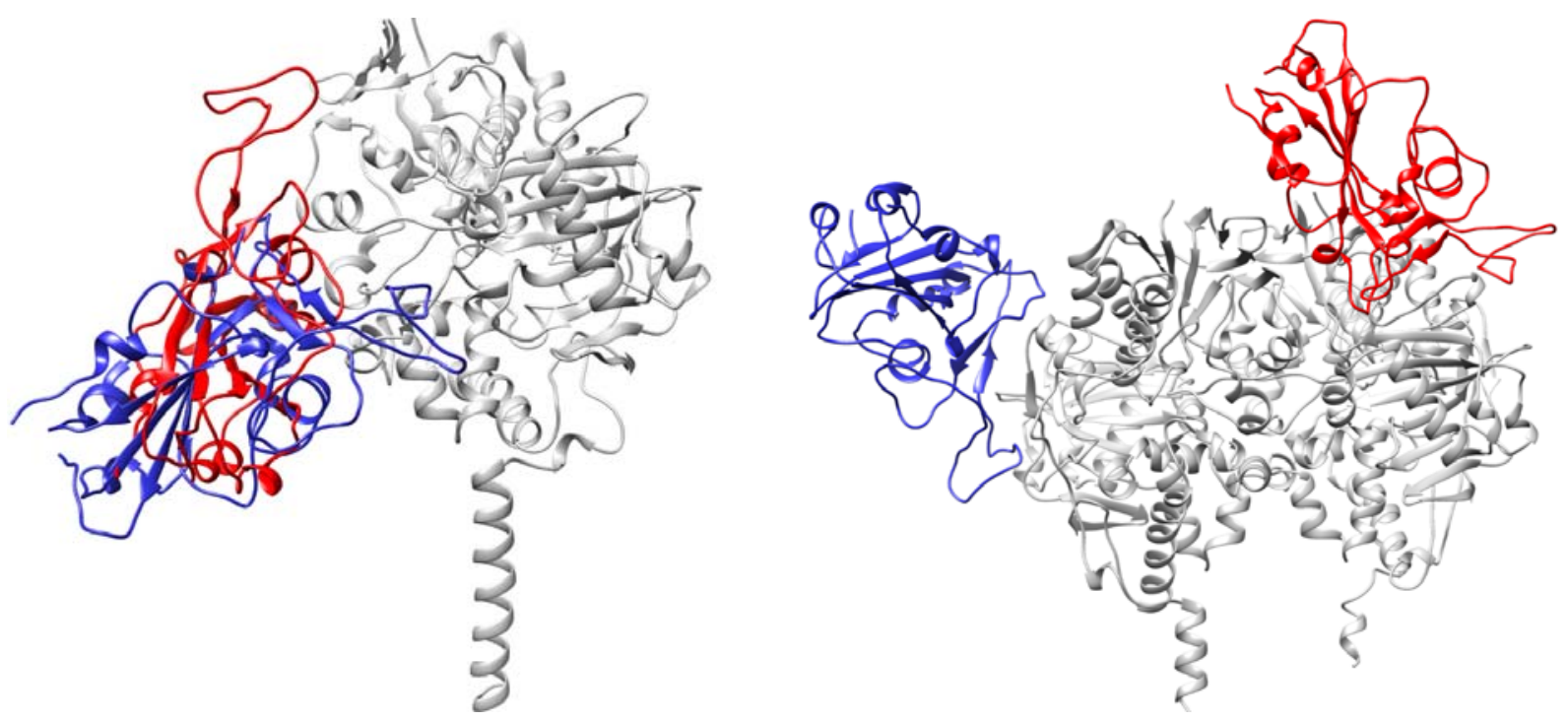

Figure 1. Overlap of the most favourable binding positions of the WT (in blue) and the SA (in red) spike protein in complex with the MAO enzymes (in gray), MAO A (left) and MAO B (right), as elucidated from molecular dynamic simulations.

As it was the case with ACE2, both SARS-CoV-2 variants predominantly interact with the MAO enzymes through their RBM region. This is evident from the fact that a majority of crucial interacting residues belongs to this structural element of the spike protein. This holds especially for the WT strain, while for the SA strain, it is observed that the mutated Asn417 residue becomes very significant in binding MAO A with a second largest individual contribution of $-4.08 \mathrm{kcal} \mathrm{mol}^{-1}$. Interestingly, the WT binds both MAOs with an almost identical exergonicity, $\Delta G_{\text {BIND }}=-38.3 \mathrm{kcal} \mathrm{mol}^{-1}$ for $M A O A$ and $\Delta G_{\text {BIND }}=-38.1 \mathrm{kcal}$ $\mathrm{mol}^{-1}$ for MAO B. Given that the stability of the WT $\cdots$ ACE2 complex was estimated at $\Delta G_{\text {BIND }}=-46.6 \mathrm{kcal}$ $\mathrm{mol}^{-1}$, a difference in a few kcals mol ${ }^{-1}$ certainly allows a formation of the matching WT ...MAO complexes. In MAO A, the interaction with the WT spike protein (S) is dominated by S-Phe486, which establishes cation $\cdots \pi$ interactions with Lys357 in MAO A (Figure S4). This is followed by S-Ser477, which joins S-Thr478 in forming hydrogen bonds with Glu329, and by S-Thr500 which interacts with the side chain carbonyl group from GIn293. It is also worth mentioning that S-Lys417 forms a salt bridge with Glu159, which imitates an analogous interaction with Asp30 from the ACE2 receptor. Considering MAO B, the relative importance in spike protein residues is reversed relative to MAO A, making S-Tyr449 the most dominant residue, which is engaged in a hydrogen bonding with $\mathrm{Gln} 49$ and in a T-shaped $\mathrm{C}-\mathrm{H} \cdots \pi$ interaction with Tyr53 in MAO B (Figure S4). Interestingly, S-Glu484, which is mutated to Lys484 in the SA strain, is the first in disfavouring binding between the proteins $\left(+1.73 \mathrm{kcal} \mathrm{mol}^{-1}\right)$. It is placed in close vicinity of the crucial MAO B residue, Arg307, yet not interacting with it, thus its unfavourable contribution. 
When a more contagious SA variant is concerned, its affinity for ACE2 is higher relative to the WT, but it is surprising that its tendency to bind both MAOs is increased as well. This is particularly interesting for MAO A, where the binding pose for the SA strain is almost identical to the one established by the WT (Figure 1), yet the affinity is increased by $10.7 \mathrm{kcal} \mathrm{mol}^{-1}$ to $\Delta G_{\text {BIND }}=-49.0 \mathrm{kcal} \mathrm{mol}^{-1}$. Recalling that its affinity for ACE2 is $\Delta G_{\text {BIND }}=-54.8 \mathrm{kcal} \mathrm{mol}^{-1}$, again only a few kcals mol ${ }^{-1}$ higher, opens a possibility that the matching SA ‥MAO A complex could be formed. There, the two crucial residues with individual contributions exceeding $4 \mathrm{kcal} \mathrm{mol}^{-1}$ are S-Leu455 and the mutated S-Asn417, which use their backbone carbonyl and side chain amide atoms, respectively, to interact with Arg297 in MAO A (Figure S5). It is worth to emphasize that all three mutations present in the SA strain are promoting MAO A binding. As mentioned, the mutated Asn417 is the second most dominant residue $\left(-4.08 \mathrm{kcal} \mathrm{mol}^{-1}\right)$, while its nonmutated analogue Lys417 in the WT has a significantly lower contribution $\left(-0.39 \mathrm{kcal} \mathrm{mol}^{-1}\right)$. Analogously, in the SA strain, Glu484 $\left(+0.07 \mathrm{kcal} \mathrm{mol}^{-1}\right)$ and Asn501 $\left(+0.51 \mathrm{kcal} \mathrm{mol}^{-1}\right)$ are replaced by Lys484 $(+0.22 \mathrm{kcal}$ $\left.\mathrm{mol}^{-1}\right)$ and Tyr501 $\left(+0.07 \mathrm{kcal} \mathrm{mol}^{-1}\right)$, respectively, from which it follows that all three mutations present in the SA variant not only promote the ACE2 binding, but also jointly promote the MAO A complex formation by $-3.98 \mathrm{kcal} \mathrm{mol}^{-1}$, which is significant.

The situation with MAO B is even more interesting. The interaction energy in the SA $\cdots M A O B$ complex is $\Delta G_{\text {BIND }}=-62.7 \mathrm{kcal} \mathrm{mol}^{-1}$, being the highest of all, even surpassing the stability of the matching SA ‥ACE2 complex by $-7.9 \mathrm{kcal} \mathrm{mol}^{-1}$. This suggests that the SA variant would, following the initial ACE2 binding and cell infiltration, mainly attach to MAO B, a process that is thermodynamically very favourable, and which might appear particularly troublesome for neurological conditions. This recognition is dominated by S-Arg346, which forms hydrogen bonds with the Glu232 side chain and the Ala35 backbone carbonyl, both from the subunit B of the MAO B enzyme (Figure S5). Such a positive pairing leads to a very high individual contribution from Arg346 $\left(-6.54 \mathrm{kcal} \mathrm{mol}^{-1}\right)$, solely contributing to around $22 \%$ of the total binding energy. One of the reasons for the high SA $\cdots$ MAO B binding affinity relative to the WT lies in different MAO B areas preferred by both strains (Figure 1). While the WT position is almost exclusively located on one subunit, the SA strain is most favourably located closer to the interface between the MAO B subunits, which allows both units to participate in the binding, and which might be, at least partly, responsible for the increased affinity. Although our analysis identified that a majority of crucial residues belongs to the subunit $B$ (Table S3), the most significant residue in MAO B is Arg242 belonging to the subunit A. Its very high individual contribution $\left(-7.35 \mathrm{kcal} \mathrm{mol}^{-1}\right)$ comes as a result of a stable salt-bridge with S-Glu340, which was persistent during MD simulations (Figure S6). Interestingly, despite such a favourable binding to $M A O B$, none of the three mutated residues emerges among those dominant for an increased complex stability. Still, all three residues make notable contributions, as the introduced Asn417 $\left(+0.06 \mathrm{kcal} \mathrm{mol}^{-1}\right)$, Lys484 $\left(-0.40 \mathrm{kcal} \mathrm{mol}^{-1}\right)$ and Tyr501 $\left(+0.08 \mathrm{kcal} \mathrm{mol}^{-1}\right)$ surpass the initial WT residues Lys417 $\left(+0.11 \mathrm{kcal} \mathrm{mol}^{-1}\right)$, Glu484 $\left(+1.73 \mathrm{kcal} \mathrm{mol}^{-1}\right)$ and Asn501 $\left(+0.16 \mathrm{kcal} \mathrm{mol}^{-1}\right)$, thus increasing the binding affinity by $-2.26 \mathrm{kcal} \mathrm{mol}^{-1}$. 
In concluding this section, let us emphasize that the affinity of both SARS-CoV-2 variants towards the MAO isoforms is very much comparable to that for their ACE2 receptor, thus indicating a feasibility and likeliness of the WT/SA $\cdots$ MAO A/B complex formation. The latter is especially supported knowing that the structural comparison of the ACE2-spike protein binding region with MAO B resulted in approximately $90 \%$ structure overlap leading to a possibility of a MAO B activity change in COVID-19 patients, despite only $51 \%$ structural similarity with the overall ACE2 structure.[33] Our results demonstrate that this recognition is particularly favourable in the case of SA $\cdots M A O B$, where the calculated binding energy surpasses that of SA $\cdots$ ACE2 by $-7.9 \mathrm{kcal} \mathrm{mol}^{-1}$, thus offering an interesting insight and perspective.

\section{Changes in the affinity of the MAO isoforms towards physiological substrates following an interaction} with the SARS-CoV-2 variants

Lastly, we evaluated how the WT/SA $\cdots$ MAO complexes impact MAO activity through the affinity for their brain amines. In doing so, we considered phenylethylamine (PEA) for both MAO isoforms, in order to place our results in the context of experimental findings by Cuperlovic-Culf, Green and co-workers,[33] and more specific amine neurotransmitters serotonin (SER) and dopamine (DOP), which are typical substrates for MAO A and MAO B, respectively. The calculated affinities are given in Table 1 and compared to relevant Michaelis-Menten constants, $K_{\mathrm{M}}$. We note in passing that in the native MAO B, both subunits revealed comparable substrate affinities without any significant preference, in line with other reports, [52] so a more exergonic binding is considered, while for the MAO B $\cdots W T / S A$ complexes, the results for both subunits are given, while we mostly discuss those pertaining to the subunits directly interacting with the spike protein.

Table 1. Changes in the binding affinity $\left(\Delta G_{\mathrm{BIND}}\right)$ between the MAO isoforms and their physiological substrates following a complex formation with the WT and SA SARS-CoV-2 variants (in kcal mol ${ }^{-1}$ ). ${ }^{\mathrm{a}, \mathrm{b}}$

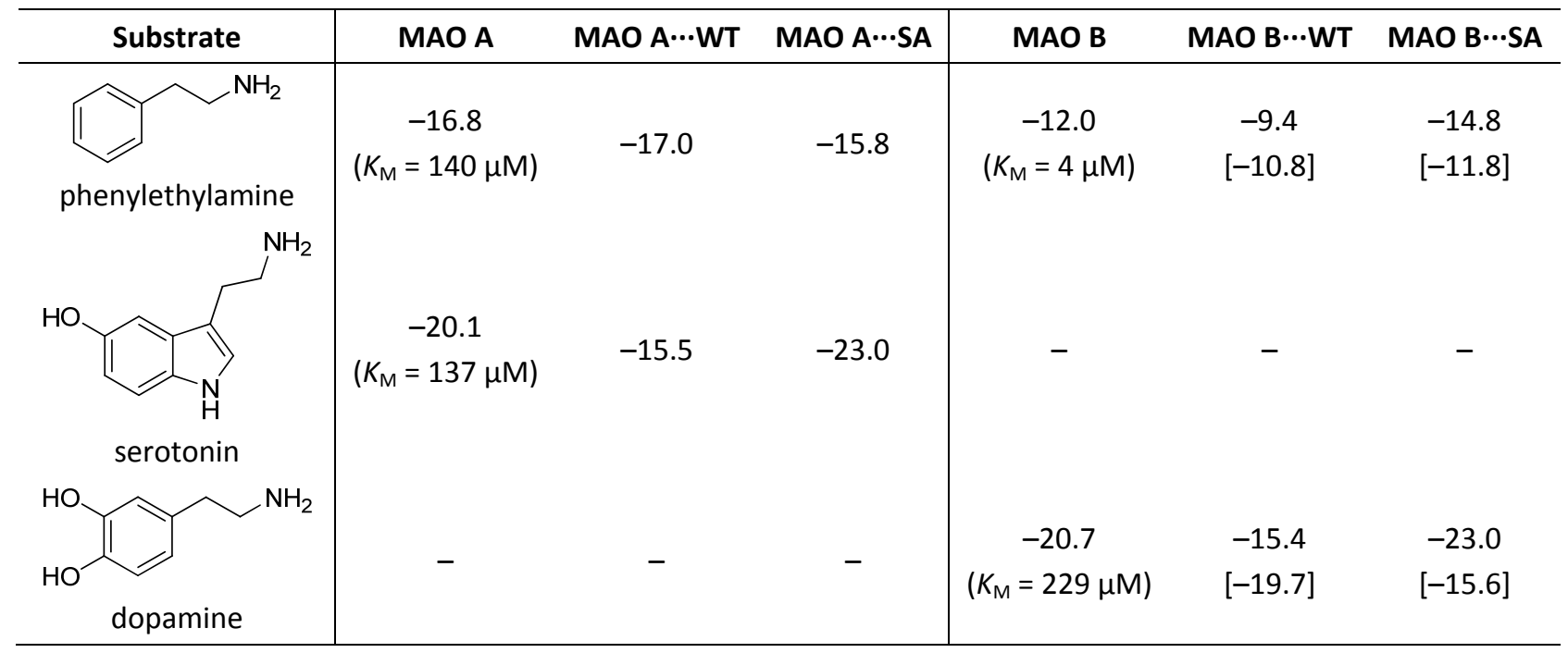

${ }^{\mathrm{a}}$ Experimental $K_{\mathrm{M}}$ values are taken from ref. [53]. ${ }^{\mathrm{b}}$ Results for the MAO B $\cdots \mathrm{WT} / \mathrm{SA}$ complexes pertain to the MAO B subunit directly interacting with the matching spike protein, while those for the other subunit are given in square brackets. 
The results for native MAOs show excellent agreement with the $K_{\mathrm{M}}$ data (Table 1 ), which lends strong credence to the employed computational setup. Specifically, PEA prefers binding to MAO A over MAO B, in line with the experimental affinities.[53] Additionally, the latter translate to a difference of 2.1 $\mathrm{kcal} \mathrm{mol}^{-1}$, which is well-matched by our computed affinity difference of $4.8 \mathrm{kcal} \mathrm{mol}^{-1}$ in favour of MAO A. Also, DOP is a better substrate for MAO B than SER is for MAO A, again tying in with experiments. There, an even stronger agreement between sets is achieved, as the experimental affinity difference of $0.3 \mathrm{kcal} \mathrm{mol}^{-1}$ between DOP and SER is almost perfectly reproduced by the computed value of $0.6 \mathrm{kcal} \mathrm{mol}^{-1}$.

When PEA is concerned, the effect of the WT on its MAO A affinity is modest, being only $0.2 \mathrm{kcal}$ $\mathrm{mol}^{-1}$ higher. Yet, for MAO B, the impact is much more pronounced, which is particularly relevant, and the

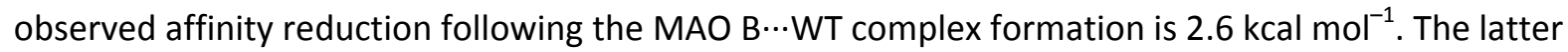
indicates about two orders of magnitude lower PEA binding, which will inevitably lead to a lower metabolic PEA conversion and higher PEA concentrations in infected individuals. This insight strongly agrees with the mentioned experiments,[33] and helps to explain a detection of lower concentrations of PEA metabolites following a COVID-19 infection, thus mimicking the effects of the irreversible MAO inhibitor selegiline, whose application also increases brain PEA levels[54] that leads to oxidative stress.[55,56] Still, we must emphasize that our results disagree with the suggestion that the spike protein is interfering with the substrate entrance into the MAO B active site.[33] The discussed binding poses in ref. [33] were obtained through docking simulations that did not explicitly consider neither the mitochondrial membrane nor the MAO B membrane bound regions,[33] which artificially allowed the WT spike protein to reside in the area close to the membrane-mediated substrate entrances,[52] which is otherwise inaccessible and occupied by the membrane. In contrast, our simulations included entire MAO structures immersed in an explicit

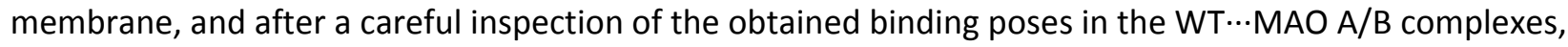
we found no evidence of the spike protein blocking the known substrate entrances.[52] Instead, based on our current results, we propose that the spike protein is interfering with the MAO activity by modifying the electrostatic environment in the complex, which impacts MAO affinity for their substrates.

A practically unchanged PEA affinity for both MAO A and the MAO A $\cdots$ WT complex comes as a result of an identical PEA binding position within MAO A in both instances (Figure2). There, its cationic amine forms a hydrogen bond with the Gln215 side chain and the carbonyl group of the FAD cofactor (Figure S7), while its ethylphenyl unit engages in a series of aromatic $\mathrm{C}-\mathrm{H} \cdots \pi$ and $\pi \cdots \pi$ stacking interactions with Tyr407, Phe352, Tyr69 and Phe208 (Table S4). Such a binding position is very much modified in MAO $B$, which is not surprising knowing that it is the predominant PEA metabolizing enzyme in the brain despite a lower affinity relative to MAO A.[53] There, PEA orients its aromatic unit towards FAD, and its cationic amine within three hydrogen bonds; with (i) the Tyr435-OH group, (ii) the Gln206 side chain, and (iii) the

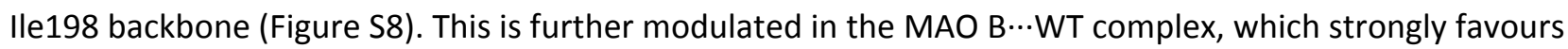
hydrophobic $\pi \cdots \pi$ stacking interactions with FAD and $\mathrm{C}-\mathrm{H} \cdots \pi$ interactions with Tyr398 and Tyr435 (Figure S9), at the expense of the hydrogen bonding contacts with $-\mathrm{NH}_{3}{ }^{+}$, which ultimately reduces the affinity. 


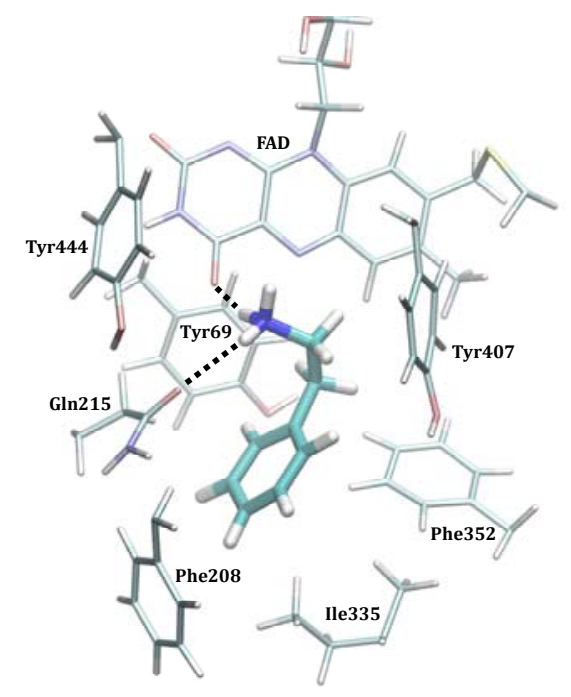

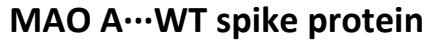

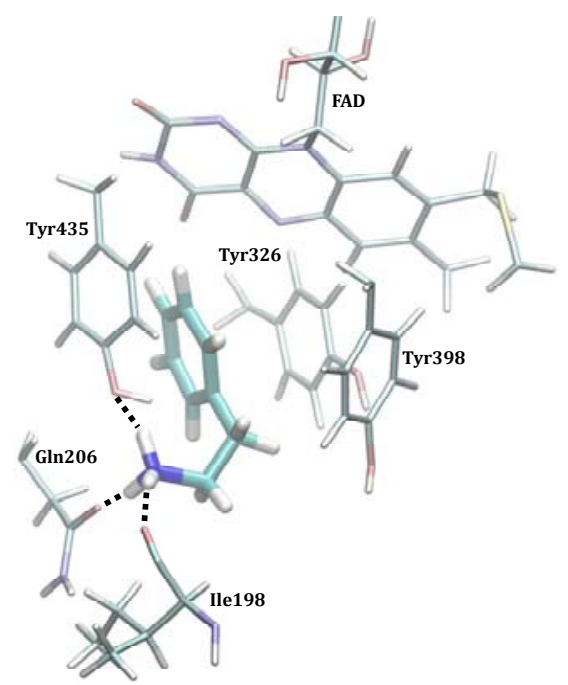

MAO B

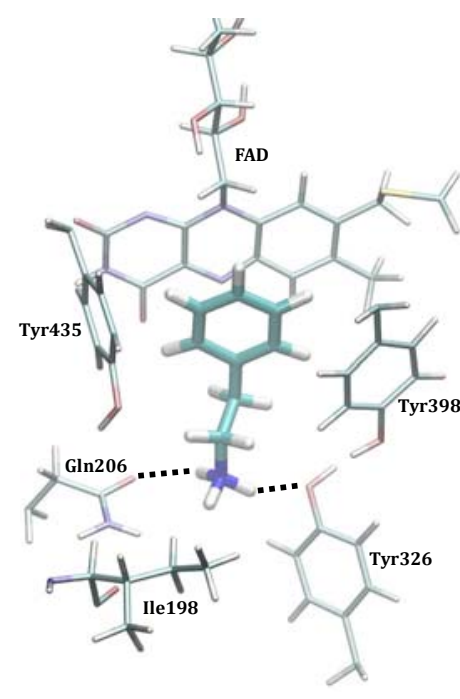

MAO B $\cdots$ WT spike protein

Figure 2. Binding position of PEA within the active sites of the MAO A $\cdots W T$ spike protein complex (left,

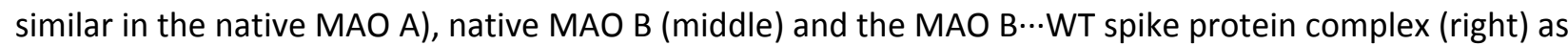
obtained from MD simulations. The results for the MAO B $\cdots$ WT complexes pertain to the MAO B subunit directly interacting with the matching spike protein.

Encouraged by rationalizing lower PEA metabolite concentrations upon the WT COVID-19 infection, we continued by analyzing the more specific MAO A and MAO B substrates, SER and DOP, respectively. SER is a typical MAO A substrate, and its affinity, $\Delta G_{\mathrm{BIND}}=-20.1 \mathrm{kcal} \mathrm{mol}^{-1}$, comes as a result of strong hydrogen bonding between its protonated amine and both the FAD carbonyl group and the GIn215 side chain amide (Figure S10), further supported by (i) the -OH hydrogen bonding with the Gly443 backbone amide and (ii) hydrophobic aromatic interactions with Tyr407 and Tyr444. This is significantly disrupted in MAO A $\cdots$ WT and results in a different binding position (Figure S11), where the mentioned three interaction motifs are replaced by the hydroxy - OH group and the protonated amine from SER interacting with the backbone amides of Asn181 and Ile207 (Figure S12), respectively; the former becoming the most dominant pairing in that case (Table S4). This results in a significantly lower SER affinity for MAO A $\cdots$ WT, being reduced by 4.6 $\mathrm{kcal} \mathrm{mol}^{-1}$ to $\Delta G_{\mathrm{BIND}}=-15.5 \mathrm{kcal} \mathrm{mol}^{-1}$. Such a significant impact inevitably leads to a lower SER metabolism upon the WT infection, which strongly corroborates experimental measurements by Shen et al.[34] On the other hand, DOP has the highest affinity among the studied amines, $\Delta G_{\mathrm{BIND}}=-20.7 \mathrm{kcal} \mathrm{mol}^{-1}$, in line with its highest $K_{\mathrm{M}}$ value of $229 \mu \mathrm{M}$. Fascinatingly, in this case, the effect of the WT strain is also the greatest, evident in a $5.3 \mathrm{kcal} \mathrm{mol}^{-1}$ reduced affinity for MAO B. The latter is supported by a notable change in DOP orientation (Figure S11), during which a range of hydrogen bonding contacts in the native MAO B (Figures S13) are replaced by mostly aromatic $\mathrm{C}-\mathrm{H} \cdots \pi$ and $\pi \cdots \pi$ stacking interactions in the complex (Figure S14). Therefore, as a conclusion, somewhat higher disturbances in the dopaminergic over serotonergic pathway could be expected following the WT variant infection, which agrees with the literature.[12,30] 


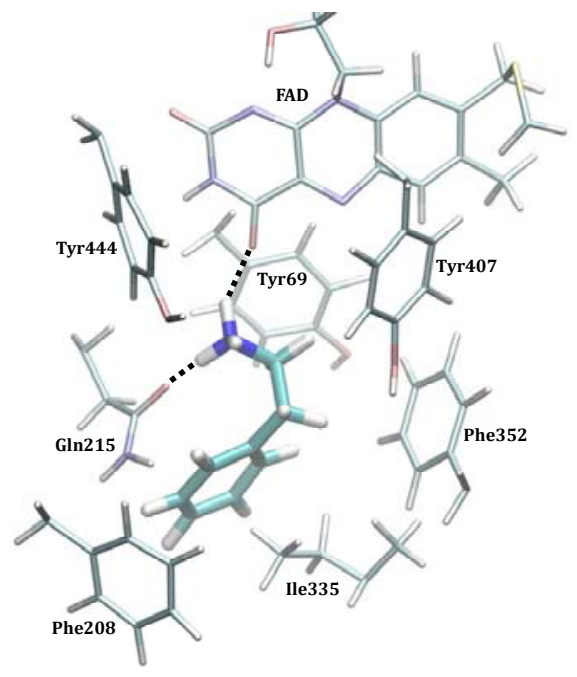

PEA in the MAO A -..SA complex

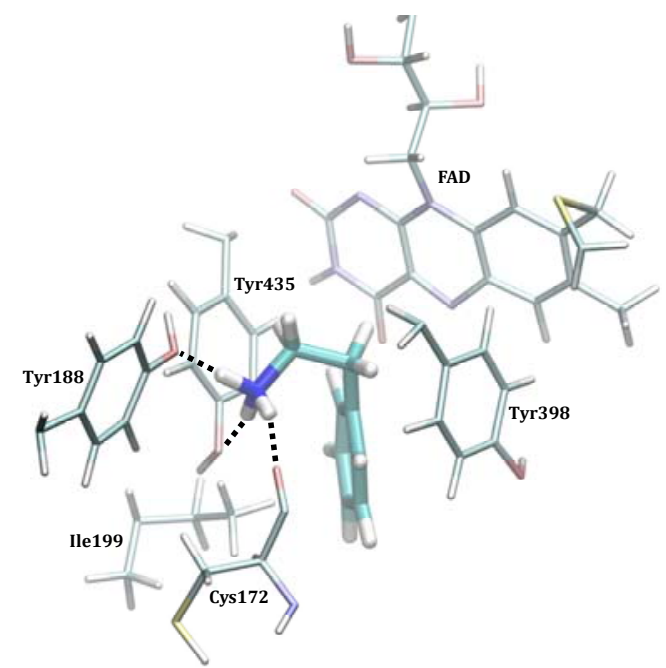

PEA in the MAO B...SA complex

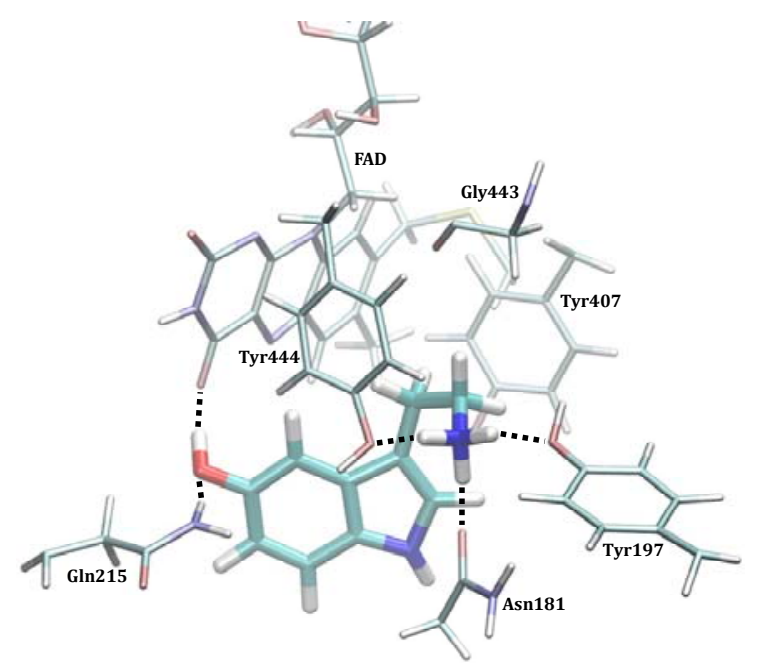

SER in the MAO A …SA complex

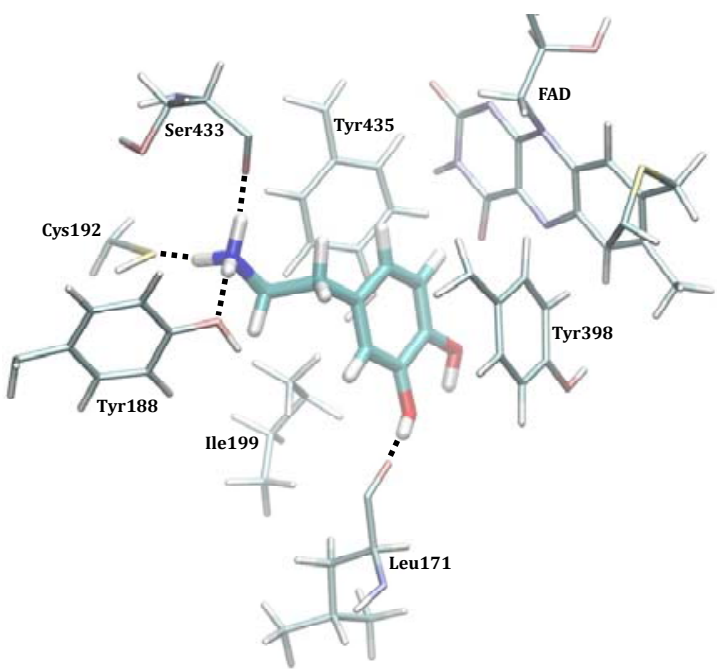

DOP in the MAO B...SA complex

Figure 3. Binding positions of substrates within the active site of both MAO isoforms following the complex formation with the SARS-CoV-2 SA variant as obtained from the MD simulations. Identification of crucial

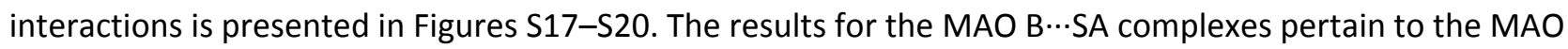
$B$ subunit directly interacting with the matching spike protein.

When a more contagious SA variant is concerned, it appears that its impact on both MAO isoforms is higher and more severe than that of the WT analogue (Figure 3, Tables S4-S5), which parallels its effect on the ACE2 receptor. Therefore, in addition to causing more disturbances in the respiratory chain, an infection with the SA strain is likely to result in more problematic outcomes for the immediate and, especially, the long-term neurological conditions. Relative to the WT, the SA strain causes the affinity of the MAO substrates to significantly increase in all cases, except for PEA and MAO A, where it is only slightly reduced, by $1.0 \mathrm{kcal} \mathrm{mol}^{-1}$ to $-15.8 \mathrm{kcal} \mathrm{mol}^{-1}$ (Table 1 ). This again suggests that PEA and MAO A are behaving differently relative to all other instances, and that the PEA pathway in the affected individuals will predominantly concern the MAO B enzyme, as it was also confirmed in the WT infection.[33] There, the 
affinity increases by $2.8 \mathrm{kcal} \mathrm{mol}^{-1}$ to $-14.8 \mathrm{kcal} \mathrm{mol}^{-1}$ (Table S5), predominantly because of favourable hydrogen bonding between its protonated amine and (i) the side chain hydroxy groups in Tyr435 and Tyr188, and (ii) the backbone amide in Cys172 (Figure S19), where the interaction with the mentioned three residues carries $56 \%$ of the total affinity. With SER, the effect of the SA infection is the largest and its affinity for MAO A increases by $2.9 \mathrm{kcal} \mathrm{mol}^{-1}$ to $-23.0 \mathrm{kcal} \mathrm{mol}^{-1}$. The latter follows after a noteworthy change in the binding position in the SA $\cdots$ MAO A complex (Figure 3), which allows SER a range of positive and persistent hydrogen bonding contacts, including those with Gln215, Tyr444, Tyr197, Asn181 and the FAD cofactor (Figure S18), where these five MAO A residues, on their own, already contribute $17.2 \mathrm{kcal}$ $\mathrm{mol}^{-1}$ to the binding energy, $75 \%$ in total, which is really striking. Such an affinity increase is analogously evident in DOP, whose affinity for MAO B becomes $2.3 \mathrm{kcal} \mathrm{mol}^{-1}$ higher and equals that for SER and MAO $A$ at $-23.0 \mathrm{kcal} \mathrm{mol}^{-1}$. This is again preceded by a different DOP binding orientation that allows it to optimize hydrogen bonding contacts with Tyr188, Ser433, Leu171 and Cys192 (Figure S20) that were all relatively insignificant for the DOP binding in MAO B and MAO B $\cdots$ WT complex, which alone are responsible for a half of the total binding energy.

The presented results raise a serious warning that, unlike a reduction in the metabolic conversion of neurotransmitters observed in the WT, the infection with the SA mutant strain will stimulate the metabolism of the investigated brain amines, which will result in their shortage. At the same time, this will increase the production of both hydrogen peroxide and thereof derived reactive oxygen species (ROS), toxic aldehydes and ammonia, which are all by-products of the MAO-catalyzed amine degradation. $[57,58]$ Unfortunately, all of the mentioned metabolites, along with the inflammation pathways, can induce neurodegenerative processes on their own and can further assist in their progression.

\section{CONCLUDING REMARKS}

A combination of docking and molecular dynamic simulations reveals that the spike protein from two SARSCoV-2 variants, namely the wild type (WT) and the mutated B.1.351 South African (SA) strain possess affinity towards the MAO enzymes that is comparable to that for its ACE2 receptor. This allows a formation

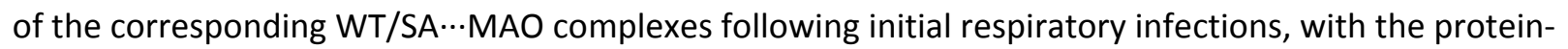
protein recognition being analogously established predominantly via residues from the receptor-binding motif in all cases. Knowing that alterations in MAO activities are a potential foundation of oxidative stress and various neuropsychiatric disorders, $[27,28,57]$ such as Parkinson's or Alzheimer's disease, $[59,60]$ the demonstrated feasibility of the WT/SA $\cdots M A O$ complex formation opens a possibility that the interference with the brain MAO activity is responsible for an increased development and faster progression of neurodegenerative illnesses in COVID-19 infected individuals; a disturbing medical issue that is presently widely discussed in the literature. 
Our computational results show that spike protein $\cdots$ MAO complexes significantly lower MAO affinities towards their neurotransmitter substrates in the WT infection, thus resulting in a reduced metabolic conversion, being firmly in line with the experimentally measured trends for PEA[33] and a range of other metabolites in mildly affected patients.[34] However, a more severe SA variant offers even more stable complexes with both MAO isoforms, which in the case of MAO B even surpasses the stability of the

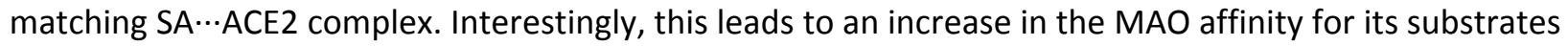
and, consequently, higher rates of their metabolic degradation, a trend that firmly agrees with experiments on serotonin and thereof derived conclusion that "serotonin levels would further decrease as the severity of COVID-19 increases".[34] The latter likely promotes neurological disturbances through the immediate overproduction of hydrogen peroxide, ROS and toxic aldehydes. In this context and within the possibility for new and more contagious mutant strains likely emerging in the near future, we firmly advise that the presented prospect for the SARS-CoV-2 induced neurological complications should be carefully monitored.

It is beyond doubt that, besides changing their enzymatic function, binding of the spike protein to the MAO enzymes can additionally alter several of their roles, such as post-translational modifications or associations with protein partners.[61] This is why a possibility the SARS-CoV-2 influences MAO activity, thereby inducing neurological complications, requires further clinical investigations, which are currently scarce since most of the ongoing research focuses on drug design. Yet, our results are, to the best of our knowledge, the first in identifying a critical role of the MAO metabolic activity in this respect, therefore placing a neurobiological link between these two conditions in the spotlight and issuing a warning that it should not be ignored. In addition, we hope our work will stimulate other researchers to identify other biological systems that could be potential targets for the spike protein,[62] which could also generate various disturbances in the infected patients. Some efforts in this direction have already been made.[63] Lastly, additional research is required to establish what effect clinically employed MAO inhibitors $[64,65]$ might have on these pathways as, currently, there is no evidence to support either the withholding or increasing MAO inhibitors in COVID-19 treatment.

\section{FUNDING}

This work was supported by CAT PHARMA (KK.01.1.1.04.0013), a project co-financed by the Croatian Government and the European Union through the European Regional Development Fund - the Competitiveness and Cohesion Operational Programme, and by the Slovenian Research Agency (P1-0012).

\section{ACKNOWLEDGMENTS}

L.H. wishes to thank the Croatian Science Foundation for a doctoral stipend through the Career Development Project for Young Researchers. We thank the Zagreb University Computing Centre for computational resources on the ISABELLA cluster. 
bioRxiv preprint doi: https://doi.org/10.1101/2021.08.30.458208; this version posted August 31,2021 . The copyright holder for this preprint (which was not certified by peer review) is the author/funder, who has granted bioRxiv a license to display the preprint in perpetuity. It is made available under aCC-BY-NC-ND 4.0 International license.

\section{REFERENCES}

[1] A. Spinelli, G. Pellino. COVID-19 pandemic: perspectives on an unfolding crisis. Br J Surg, 2020, 107, 785-787.

[2] E. Dong, H. Du, L. Gardner. An interactive web-based dashboard to track COVID-19 in real time. Lancet Infect Dis, 2020, 20, 533-534.

[3] K. Yuki, M. Fujiogi, S. Koutsogiannaki. COVID-19 pathophysiology: A review. Clin Immunol, 2020, 215, 108427.

[4] M. Nicola, Z. Alsafi, C. Sohrabi, A. Kerwan, A. Al-Jabir, C. losifidis, M. Agha, R. Agha. The socioeconomic implications of the coronavirus pandemic (COVID-19): A review. Int J Surg, 2020, 78, 185193.

[5] M. Taquet, J. R. Geddes, M. Husain, S. Luciano, P. J. Harrison. 6-month neurological and psychiatric outcomes in 236379 survivors of COVID-19: a retrospective cohort study using electronic health records. Lancet Psychiat, 2021, 8, 416-427.

[6] M. A. Ellul, L. Benjamin, B. Singh, S. Lant, B. D. Michael, A. Easton, R. Kneen, S. Defres, J. Sejvar, T. Solomon. Neurological associations of COVID-19. Lancet Neurol, 2020, 19, 767-783.

[7] A. S. Zubair, L. S. McAlpine, T. Gardin, S. Farhadian, D. E. Kuruvilla, S. Spudich. Neuropathogenesis and neurologic manifestations of the coronaviruses in the age of coronavirus disease 2019: A review. JAMA Neurol, 2020, 77, 1018-1027.

[8] A. Lippi, R. Domingues, C. Setz, T. F. Outeiro, A. Krisko. SARS-CoV-2: at the crossroad between aging and neurodegeneration. Mov Disord, 2020, 35, 716-720.

[9] S. Natoli, V. Oliveira, P. Calabresi, L. F. Maia, A. Pisani. Does SARS-Cov-2 invade the brain? Translational lessons from animal models. Eur J Neurol, 2020, 27, 1764-1773.

[10] P. Zhou, X.-L. Yang, X.-G. Wang, B. Hu, L. Zhang, W. Zhang, H.-R. Si, Y. Zhu, B. Li, C.-L. Huang, H.-D. Chen, J. Chen, Y. Luo, H. Guo, R.-D. Jiang, M.-Q. Liu, Y. Chen, X.-R. Shen, X. Wang, X.-S. Zheng, K. Zhao, Q.-J. Chen, F. Deng, L.-L. Liu, B. Yan, F.-X. Zhan, Y.-Y. Wang, G.-F. Xiao, Z.-L. Shi. A pneumonia outbreak associated with a new coronavirus of probable bat origin. Nature, 2020, 579, 270-273.

[11] I. Hamming, W. Timens, M. L. C. Bulthuis, A. T. Lely, G. J. Navis, H. van Goor. Tissue distribution of ACE2 protein, the functional receptor for SARS coronavirus. A first step in understanding SARS pathogenesis. J Pathol, 2004, 203, 631-637.

[12] S. Nataf. An alteration of the dopamine synthetic pathway is possibly involved in the pathophysiology of COVID-19. J Med Virol, 2020, 92, 1743-1744.

[13] N. Poyiadji, G. Shahin, D. Noujaim, M. Stone, S. Patel, B. Griffith. COVID-19-associated acute hemorrhagic necrotizing encephalopathy: Imaging features. Radiology, 2020, 296, E119-E120.

[14] K. Li, C. Wohlford-Lenane, S. Perlman, J. Zhao, A. K. Jewell, L. R. Reznikov, K. N. Gibson-Corley, D. K. Meyerholz, P. B. McCray Jr. Middle east respiratory syndrome coronavirus causes multiple organ damage and lethal disease in mice transgenic for human dipeptidyl peptidase 4. J Infect Dis, 2016, $213,712-722$. 
[15] M. K. M. Karuppan, D. Devadoss, M. Nair, H. S. Chand, M. K. Lakshmana. SARS-CoV-2 Infection in the Central and Peripheral Nervous System-Associated Morbidities and Their Potential Mechanism. Mol Neurobiol, 2021, 58, 2465-2480.

[16] M. V. Putilina, D. V. Grishin. SARS-CoV-2 (COVID-19) as a Predictor of Neuroinflammation and Neurodegeneration: Potential Treatment Strategies. Neurosci Behav Physi, 2021, 51, 577-582.

[17] M. Dolatshahi, M. Sabahi, M. H. Aarabi. Pathophysiological Clues to How the Emergent SARS-CoV-2 Can Potentially Increase the Susceptibility to Neurodegeneration. Mol Neurobiol, 2021, 58, 23792394.

[18] E. A. Kiyatkin. Respiratory depression and brain hypoxia induced by opioid drugs: morphine, oxycodone, heroin, and fentanyl. Neuropharmacology, 2019, 151, 219-226.

[19] L. Onofrio, M. Caraglia, G. Facchini, V. Margherita, S. De Placido, C. Buonerba. Toll-like receptors and COVID-19: a two-faced story with an exciting ending. Future Sci OA, 2020, 6, FSO605.

[20] C. Bai, A. Warshel. Critical Differences between the Binding Features of the Spike Proteins of SARSCoV-2 and SARS-CoV. J Phys Chem B, 2020, 124, 5907-5912.

[21] A. Spinello, A. Saltalamacchia, A. Magistrato. Is the Rigidity of SARS-CoV-2 Spike Receptor-Binding Motif the Hallmark for Its Enhanced Infectivity? Insights from All-Atom Simulations. J Phys Chem Lett, 2020, 11, 4785-4790.

[22] M. Desforges, A. Le Coupanec, P. Dubeau, A. Bourgouin, L. Lajoie, M. Dubé, P. J. Talbot. Human coronaviruses and other respiratory viruses: underestimated opportunistic pathogens of the central nervous system? Viruses, 2019, 12, 14.

[23] Y. Wu, X. Xu, Z. Chen, J. Duan, K. Hashimoto, L. Yang, C. Liu, C. Yanga. Nervous system involvement after infection with COVID-19 and other coronaviruses. Brain Behav Immun, 2020, 87, 18-22.

[24] S. Khan, R. Siddique, H. Li, A. Ali, M. A. Shereen, N. Bashir, M. Xue. Impact of coronavirus outbreak on psychological health. J Glob Health, 2020, 10, 010331.

[25] H. Vlajinac, S. Sipetic, J. Marinkovic, I. Ratkov, J. Maksimovic, E. Dzoljic, V. Kostic. The stressful life events and Parkinson's disease: a case-control study. Stress Health, 2013, 29, 50-55.

[26] K. F. Tipton. 90 years of monoamine oxidase: some progress and some confusion. J Neural Transm (Vienna), 2018, 125, 1519-1551.

[27] D. N. Jones, M. A. Raghanti. The role of monoamine oxidase enzymes in the pathophysiology of neurological disorders. J Chem Neuroanat, 2021, 114, 101957.

[28] Y. Santin, J. Resta, A. Parini, J. Mialet-Perez. Monoamine oxidases in age-associated diseases: New perspectives for old enzymes. Ageing Res Rev, 2021, 66, 101256.

[29] I. O. Awogbindin, B. Ben-Azu, B. A. Olusola, E. T. Akinluyi, P. A. Adeniyi, T. Di Paolo, M.-È. Tremblay. Microglial Implications in SARS-CoV-2 Infection and COVID-19: Lessons From Viral RNA Neurotropism and Possible Relevance to Parkinson's Disease. Front Cell Neurosci, 2021, 15, 670298.

[30] L. Attademo, F. Bernardini. Are dopamine and serotonin involved in COVID-19 pathophysiology? Eur J Psychiatry, 2021, 35, 62-63. 
[31] G. Abate, M. Memo, D. Uberti. Impact of COVID-19 on Alzheimer's disease risk: Viewpoint for research action. Healthcare (Basel), 2020, 8, 286.

[32] T. Schirinzi, D. Landi, C. Liguori. COVID-19: dealing with a potential risk factor for chronic neurological disorders. J Neurol, 2021, 268, 1171-1178.

[33] M. Cuperlovic-Culf, E. L. Cunningham, H. Teimoorinia, A. Surendra, X. Pan, S. A. L. Bennett, M. Jung, B. McGuiness, A. P. Passmore, D. Beverland, B. D. Green. Metabolomics and computational analysis of the role of monoamine oxidase activity in delirium and SARS-COV-2 infection. Sci Rep, 2021, 11, 10629.

[34] B. Shen, X. Yi, Y. Sun, X. Bi, J. Du, C. Zhang, S. Quan, F. Zhang, R. Sun, L. Qian, W. Ge, W. Liu, S. Liang, H. Chen, Y. Zhang, J. Li, J. Xu, Z. He, B. Chen, J. Wang, H. Yan, Y. Zheng, D. Wang, J. Zhu, Z. Kong, Z. Kang, X. Liang, X. Ding, G. Ruan, N. Xiang, X. Cai, H. Gao, L. Li, S. Li, Q. Xiao, T. Lu, Y. Zhu, H. Liu, H. Chen, T. Guo. Proteomic and Metabolomic Characterization of COVID-19 Patient Sera. Cell, 2020 , $182,59-72$.

[35] J. Lan, J. Ge, J. Yu, S. Shan, H. Zhou, S. Fan, Q. Zhang, X. Shi, Q. Wang, L. Zhang, X. Wang. Structure of the SARS-CoV-2 spike receptor-binding domain bound to the ACE2 receptor. Nature, 2020, 581, 215220.

[36] R. Yan, Y. Zhang, Y. Li, L. Xia, Y. Guo, Q. Zhou. Structural basis for the recognition of SARS-CoV-2 by full-length human ACE2. Science, 2020, 367, 1444-1448.

[37] Y. Wang, M. Liu, J. Gao. Enhanced receptor binding of SARS-CoV-2 through networks of hydrogenbonding and hydrophobic interactions. Proc Natl Acad Sci U S A, 2020, 117, 13967-13974.

[38] S. Piplani, P. K. Singh, D. A. Winkler, N. Petrovsky. In silico comparison of SARS-CoV-2 spike proteinACE2 binding affinities across species and implications for virus origin. Sci Rep, 2021, 11, 13063.

[39] E. N. Muratov, R. Amaro, C. H. Andrade, N. Brown, S. Ekins, D. Fourches, O. Isayev, D. Kozakov, J. L. Medina-Franco, K. M. Merz, T. I. Oprea, V. Poroikov, G. Schneider, M. H. Todd, A. Varnek, D. A. Winkler, A. V. Zakharov, A. Cherkasov, A. Tropsha. A critical overview of computational approaches employed for COVID-19 drug discovery. Chem Soc Rev, 2021, in print. DOI: 10.1039/D0CS01065K

[40] M. Ramanathan, I. D. Ferguson, W. Miao, P. A. Khavarib. SARS-CoV-2 B.1.1.7 and B.1.351 spike variants bind human ACE2 with increased affinity. Lancet Infect Dis, 2021, 21, 1070.

[41] E. Boehm, I. Kronig, R. A. Neher, I. Eckerle, P. Vetter, L. Kaiser. Novel SARS-CoV-2 variants: the pandemics within the pandemic. Clin Microbiol Infect, 2021, 27, 1109-1117.

[42] B. J. Williams-Noonan, N. Todorova, K. Kulkarni, M.-I. Aguilar, I. Yarovsky. An Active Site Inhibitor Induces Conformational Penalties for ACE2 Recognition by the Spike Protein of SARS-CoV-2.J Phys Chem B, 2021, 125, 2533-2550.

[43] N. A. Murugan, P. S. Javali, C. J. Pandian, M. A. Ali, V. Srivastava, J. Jeyaraman. Computational Investigation of Increased Virulence and Pathogenesis of SARS-CoV-2 Lineage B.1.1.7. DOI: 10.1101/2021.01.25.428190

[44] Y. Huang, C. Yang, X.-F. Xu, W. Xu, S.-W. Liu. Structural and functional properties of SARS-CoV-2 spike protein: potential antivirus drug development for COVID-19. Acta Pharmacol Sin, 2020, 41, 11411149. 
[45] B. Bakhshandeh, S. G. Sorboni, A.-R. Javanmard, S. S. Mottaghi, M.-R. Mehrabi, F. Sorouri, A. Abbasi, Z. Jahanafrooz. Variants in ACE2; potential influences on virus infection and COVID-19 severity. Infect Genet Evol, 2021, 90, 104773.

[46] H. Tegally, E. Wilkinson, M. Giovanetti, A. Iranzadeh, V. Fonseca, J. Giandhari, D. Doolabh, S. Pillay, E. J. San, N. Msomi, K. Mlisana, A. von Gottberg, S. Walaza, M. Allam, A. Ismail, T. Mohale, A. J. Glass, S. Engelbrecht, G. Van Zyl, W. Preiser, F. Petruccione, A. Sigal, D. Hardie, G. Marais, N.-Y. Hsiao, S. Korsman, M.-A. Davies, L. Tyers, I. Mudau, D. York, C. Maslo, D. Goedhals, S. Abrahams, O. LagudaAkingba, A. Alisoltani-Dehkordi, A. Godzik, C. K. Wibmer, B. T. Sewell, J. Lourenço, L. C. Jr Alcantara, S. L. Kosakovsky Pond, S. Weaver, D. Martin, R. J. Lessells, J. N. Bhiman, C. Williamson, T. de Oliveira. Detection of a SARS-CoV-2 variant of concern in South Africa. Nature, 2021, 592, 438-443.

[47] A. Winger, T. Caspari. The Spike of Concern-The Novel Variants of SARS-CoV-2. Viruses, 2021, 13, 1002.

[48] Z. Wang, F. Schmidt, Y. Weisblum, F. Muecksch, C. O. Barnes, S. Finkin, D. Schaefer-Babajew, M. Cipolla, C. Gaebler, J. A. Lieberman, T. Y. Oliveira, Z. Yang, M. E. Abernathy, K. E. Huey-Tubman, A. Hurley, M. Turroja, K. A. West, K. Gordon, K. G. Millard, V. Ramos, J. Da Silva, J. Xu, R. A. Colbert, R. Patel, J. Dizon, C. Unson-O'Brien, I. Shimeliovich, A. Gazumyan, M. Caskey, P. J. Bjorkman, R. Casellas, T. Hatziioannou, P. D. Bieniasz, M. C. Nussenzweig. mRNA vaccine-elicited antibodies to SARS-CoV-2 and circulating variants. Nature, 2021, 592, 616-622.

[49] H. Gu, Q. Chen, G. Yang, L. He, H. Fan, Y.-Q. Deng, Y. Wang, Y. Teng, Z. Zhao, Y. Cui, Y. Li, X.-F. Li, J. Li, N.-N. Zhang, X. Yang, S. Chen, Y. Guo, G. Zhao, X. Wang, D.-Y. Luo, H. Wang, X. Yang, Y. Li, G. Han, Y. $\mathrm{He}, \mathrm{X}$. Zhou, S. Geng, X. Sheng, S. Jiang, S. Sun, C.-F. Qin, Y. Zhou. Adaptation of SARS-CoV-2 in BALB/C mice for testing vaccine efficacy. Science, 2020, 369, 1603-1607.

[50] H. Liu, Q. Zhang, P. Wei, Z. Chen, K. Aviszus, J. Yang, W. Downing, C. Jiang, B. Liang, L. Reynoso, G. P. Downey, S. K. Frankel, J. Kappler, P. Marrack, G. Zhang. The basis of a more contagious 501Y.V1 variant of SARS-CoV-2. Cell Res, 2021, 31, 720-722.

[51] N. Homeyer, H. Gohlke. Free Energy Calculations by the Molecular Mechanics Poisson-Boltzmann Surface Area Method. Mol Inform, 2012, 31, 114-122.

[52] H. B. L. Jones, R. M. Crean, A. Mullen, E. G. Kendrick, S. D. Bull, S. A. Wells, D. R. Carbery, F. MacMillan, M. W. van der Kamp, C. R. Pudney. Exposing the Interplay Between Enzyme Turnover, Protein Dynamics, and the Membrane Environment in Monoamine Oxidase B. Biochemistry, 2019, $58,2362-2372$.

[53] M. B. H. Youdim, D. Edmondson, K. F. Tipton. The therapeutic potential of monoamine oxidase inhibitors. Nat Rev Neurosci, 2006, 7, 295-309.

[54] G. P. Reynolds, P. Riederer, M. Sandler, K. Jellinger, D. Seemann. Amphetamine and 2phenylethylamine in post-mortem Parkinsonian brain after (-)deprenyl administration. J Neural Transm, 1978, 43, 271-277.

[55] D. Pregeljc, D. Teodorescu-Perijoc, R. Vianello, N. Umek, J. Mavri. How important is the use of cocaine and amphetamines in the development of Parkinson disease? A computational study. Neurotox Res, 2020, 37, 724-731.

[56] N. Umek, B. Geršak, N. Vintar, M. Šoštarič, J. Mavri. Dopamine autoxidation is controlled by acidic pH. Front Mol Neurosci, 2018, 11, 467. 
[57] M. Pavlin, M. Repič, R. Vianello, J. Mavri. The chemistry of neurodegeneration: kinetic data and their implications. Mol Neurobiol, 2016, 53, 3400-3415.

[58] R. Vianello, C. Domene, J. Mavri. The use of multiscale molecular simulations in understanding a relationship between the structure and function of biological systems of the brain: The application to monoamine oxidase enzymes. Front Neurosci, 2016, 10, 24.

[59] P. Riederer, T. Müller. Monoamine oxidase-B inhibitors in the treatment of Parkinson's disease: clinical-pharmacological aspects. J Neural Transm (Vienna), 2018, 125, 1751-1757.

[60] S. Manzoor, N. Hoda. A comprehensive review of monoamine oxidase inhibitors as Anti-Alzheimer's disease agents: A review. Eur J Med Chem, 2020, 206, 112787.

[61] M. Salmi, S. Jalkanen. VAP-1: An adhesin and an enzyme. Trends Immunol, 2001, 22, 211-216.

[62] G. Galindez, J. Matschinske, T. D. Rose, S. Sadegh, M. Salgado-Albarrán, J. Späth, J. Baumbach, J. K. Pauling. Lessons from the COVID-19 pandemic for advancing computational drug repurposing strategies. Nat Comput Sci, 2021, 1, 33-41.

[63] D. Idrees, V. Kumar. SARS-CoV-2 spike protein interactions with amyloidogenic proteins: Potential clues to neurodegeneration. Biochem Biophys Res Commun, 2021, 554, 94-98.

[64] T. Tandarić, R. Vianello. Computational insight into the mechanism of the irreversible inhibition of monoamine oxidase enzymes by the anti-parkinsonian propargylamine inhibitors rasagiline and selegiline. ACS Chem Neurosci, 2019, 10, 3532-3542.

[65] M. Ostadkarampour, E. E. Putnins. Monoamine Oxidase Inhibitors: A Review of Their AntiInflammatory Therapeutic Potential and Mechanisms of Action. Front Pharmacol, 2021, 12, 676239. 